\title{
Topicalization effects in cued recall of technical prose
}

\author{
DAVID E. KIERAS \\ University of Arizona, Tucson, Arizona 85721
}

\begin{abstract}
The perceived topic of a passage should determine what information is given priority in storage effort for later recall. The topic should also determine how effective a later recall cue should be, in that recall should be best if the cue is the same as the passage topic. These issues were studied by investigating cued recall of passages that contained information about two candidate topic items, either of which could be marked as the passage topic by initial mention or the sentence surface subject position. The recall cue was either the item marked as the topic or the nontopic item. If the cue was the topic, recall about the topic item was greater than recall about the nontopic item. If the cue was the nontopic item, recall about the two items was roughly the same, unaffected by the topic marking. But the topic and nontopic cues produced the same overall level of recall. In contrast to the original hypothesis, the results are interpreted as the topic marking and recall cue acting as instructions for what information the subject should emphasize in recall. It is argued that the two-topic passages used in this work are processed differently from the usual one-topic passages in prose memory studies.
\end{abstract}

Some of the information in a piece of prose is more important than the rest of the information. If one is asked to study a passage, one should remember the more important information better than the less important information. This fact is well documented under the name of the "levels effect," in which information at a high level of importance in a propositional analysis of a passage is better recalled than information at low levels (see Johnson, 1970; Kintsch \& Keenan, 1973; Kintsch, Kozminsky, Streby, McKoon, \& Keenan, 1975; Kozminsky, 1977; Meyer, 1977). The importance level is defined in terms of the hierarchical semantic structure of the passage information.

The role that importance level plays in current theory of prose memory is best represented by the macrostructure theory of comprehension (Kintsch, 1977; Kintsch \& van Dijk, 1978; van Dijk, 1977). According to this theory, a reader selects or constructs a set of macrostructure propositions that represents the gist or important content of the passage, and which is then given priority for storage into memory. The levels effect appears because propositions higher in the passage structure will usually be important macropropositions, or closely related to the macropropositions, and so are likely to be stored and later recalled by the reader.

Although the semantic content of a passage is the main determinant of which propositions are important,

This research was supported by the Personnel and Training Research Programs, Office of Naval Research, under Contract N00014-78-C-0509, Contract Authority Identification No. NR 157-423. Thanks are due to Gary Foulke, Susan Bovair, Mark Stempski, Jim Lentz, and Kathy Warrick for their assistance in collecting and analyzing the data. Requests for reprints should be sent to the author at the Department of Psychology, University of Arizona, Tucson, Arizona 85721. there also exist many features of textual surface structure that signal what content is important, or thematic (van Dijk, 1979), and so should influence what is remembered from a passage. One such signal is a title for the passage. Kozminsky (1977), using passages with two alternate themes, found that recall was biased in favor of propositions relevant to the theme mentioned in the title. Another important surface-level signal is initial mention; items or ideas appearing first in a passage tend to be viewed as thematically important simply by virtue of their position (Kieras, 1980). Better recall of initially appearing information has been reported (e.g., Kintsch et al., 1975; Meyer, 1977), but it has usually been attributed to better recall of important information, which in conformity with a linguistic convention, usually appears first. However, such studies have usually confounded structural level of importance with initial mention. Another important surface-level signal to thematic content is the topic-comment assignment of items mentioned in individual sentences (Perfetti \& Goldman, 1974, 1975; van Dijk, 1979). The simplest form of this signal is simply which item appears as the surface subject of the passage sentences. Kieras (1981) found that a particular candidate topic was chosen as the passage topic more often if it appeared as the surface subject of the sentences. Clements (1979), using sentence topic-comment assignment and other aspects of assigning thematic importance, found that material marked as important was recalled better than unmarked material.

Such signals should govern what is stored. Now consider how readers might retrieve information from a previously studied passage when given a cue to the content. The obvious application of macrostructure theory is that the macrostructure of the passage deter- 
mines not only what is to be stored, but also what the retrieval routes for that information might be. That is, the reader selects the main item, or main referent, of the passage, around which the macrostructure should be organized (see Kieras, 1980; van Dijk, 1979), and uses this perceived topic as a sort of "address" for storage. When given a cue for recall, the reader looks first under the corresponding "address" in memory for the passage information. If the desired information is found, recall proceeds smoothly. If not, the retrieval attempt will be disrupted and a different retrieval strategy must be used, resulting in poorer recall. Hence the originally perceived topic of a passage will influence the effectiveness of a recall cue; a cue that matches the topic should be superior to one that mismatches. Such an effect would be a prose memory analogue to the "encoding-specificity" principle studied heavily in verbal learning.

The two studies reported here address the role of topicalization in cued recall using brief technical passages. The question is whether marking an item as the passage topic and cuing recall with either the topic item or a nontopic item affects recall for propositions about passage items. Experiment 1 demonstrates effects using initial mention as the topicalization marker. Experiment 2 follows up by exploring these effects in more detail, using initial mention and surface subject assignment as topicalization markers and examining recall for passages in which the topic was determined by semantic factors rather than textual surface structure. An important feature of these experiments is that the topic marking was done independently of the specific passage content. Thus recall of exactly the same material can be compared when it is marked as a topic and when it is unmarked. This was achieved by using passages chosen from earlier studies (Kieras, 1980, Note 1) that each had two candidate topics, either of which could be marked as the passage topic. Recall was compared for cases in which the different candidate topics were marked as the passage topic and cases in which the different candidate topics served as the recall cue.

\section{EXPERIMENT 1}

In this experiment, several two-topic passages were used, with the topic marking produced by the candidate topic that was described first in the passage (see Kieras, 1980). An example passage is shown in Table 1, in which the two candidate topic items, labeled A and B, are "Biotransformation" and "The Liver." The passage consists of two sentences about Item A, two sentences about Item $B$, and a linking sentence that connects them. The A-first version consists of the two A sentences, followed by the linking sentence, followed by the two B sentences. In the B-first version, the B sentences appear first, then the linking sentence, and the A sentences. The passages were prepared to be reasonably comprehensible in both versions.
Table 1

A-First and B-First Versions of an Example Two-Topic Passage

A-First Version

Biotransformation is the chemical transformation that causes the inactivation of drugs, the detoxification of environmental pollutants, and the deactivation of chemicals that can cause cancer. Biotransformation of harmful agents involves an oxidation reaction which is mediated by complex enzymes, and if this process does not take place, a drug entering the body may act indefinitely. Biotransformation defends the body against the effects of toxins and is carried out in the liver. The liver, weighing three pounds in the human adult, is the largest organ in the body and performs diverse functions. Through the large portal vein of the liver passes all the blood that has absorbed digested food and other substances from the intestines.

\section{B-First Version}

The liver, weighing three pounds in the human adult, is the largest organ in the body and performs diverse functions. Through the large portal vein of the liver passes all the blood that has absorbed digested food and other substances from the intestines. Biotransformation defends the body against the effects of toxins and is carried out in the liver. Biotransformation is the chemical transformation that causes the inactivation of drugs, the detoxification of environmental pollutants, and the deactivation of chemicals that can cause cancer. Biotransformation of harmful agents involves an oxidation reaction which is mediated by complex enzymes, and if this process does not take place, a drug entering the body may act indefinitely.

\section{Method}

Materials. Eight passages were selected from those reported in Kieras (Note 1, Experiment 4) that showed good effects of the initial-mention variable. These passages would thus be expected to produce different apparent topics when presented in the two orders. For each passage, two cues, one or two words long, were devised that named each candidate topic of the passages. The passages were prepared as small booklets, one for each subject, with one passage per page.

Design. The experiment was within subjects, with four conditions, corresponding to the two passage versions, A-first or B-first, combined with the two possible cues, the $A$ cue and the B cue. A series of 8 by 8 random Latin squares was used to determine the assignment of passages to version and cue conditions for each subject, ensuring that for multiples of eight subjects, each passage would appear twice in each condition and each subject would see each condition twice and each passage once. Another series of Latin squares was used to balance the order of appearance in the booklets.

Subjects. Forty-ight subjects of both sexes participated for extra credit in an introductory psychology course. The session required about $1 \mathrm{~h}$.

Procedure. The subjects were run in groups of up to 20 in size. The subjects studied each passage in their booklets; they were paced by the experimenter at $1 \mathrm{~min} /$ passage. After reading all eight passages, subjects immediately began working on a distractor task consisting of a large sheet of arithmetic problems. After $8 \mathrm{~min}$ had elapsed, subjects were instructed on the recall procedure. Each subject was given a booklet consisting of eight pages, with a cue word for one of the passages at the top of each page. The recall cues were arranged in the same order as the passages for each subject. The subjects were instructed to write as much as they could remember, in their own words, of the passage corresponding to the cue word. They were paced at $3 \mathrm{~min}$ for each passage recall attempt. The amount of time allowed was observed to be adequate. 


\section{Results}

A propositional analysis was performed on the passages and used as a scoring key, along the lines described by Kintsch (1974), Bovair and Kieras (Note 2), and Turner and Greene (Note 3). The recall protocols were scored blind, without knowledge of the original passage version condition, for reproductions of the passage propositions. The scoring criterion was fairly strict; a proposition was counted as recalled only if the subject supplied all of the same terms in the proposition, with synonymous terms or references allowed. No credit was given for partial recall of a proposition or for general similarity of meaning. The strict scoring, together with the delayed-recall procedure, produced a fairly low level of recall, around $11 \%$ on the average. Since such low levels of recall are difficult to score, it was desirable to have some check on the reliability of the scoring. Two independent raters scored the recall protocols, each using his own propositional analysis, and then the rater was included as a factor in the analysis. Recalled propositions from the sentences about each candidate topic item were then counted, but the propositions from the linking sentence were not included. Hence the analyses were done only on the recall of propositions from the two sentences about each item. The amount of recall was converted to proportions to eliminate the slight differences in the number of propositions contained in the sentences, averaged over passages, and subjected to an ANOVA. The factors in the analysis were the scoring rater, the item of recall (the topicalized item or the nontopicalized item), the recall cue (topic or nontopic), and the passage version (A or B). In these experiments, the materials were not randomly sampled, but they were carefully constructed and selected to produce the intended topic manipulation. Hence passages were not treated as a random factor (see Clark, Cohen, Smith, \& Keppel, 1976; Wike \& Church, 1976).

With regard to scoring reliability, the correlation was .861 between mean recall scores of the two raters, using the 384 points corresponding to mean recall of each item from each passage for each subject in each condition. The second rater produced scores that were very slightly higher than those of the first rater $(10.6 \%$ vs. $11.3 \%$, which was marginally significant $[F(1,47)=$ $3.25, \mathrm{p}<.1]$. Hence there was very good agreement between the two raters, especially considering that they used separate propositional analyses. But there was an effect of rater, which will be discussed below.

The mean proportions of recall of each item in each condition are shown in Table 2. The main effect of item of recall was significant $[F(1,47)=14.82, p<.01]$ propositions from sentences about the topic items were recalled better overall than those about nontopic items $(13 \%$ vs. $9 \%)$. But there was an interaction of item of recall with cue, which was strongly significant $[F(1,47)$ $=34.18 ; \mathrm{p}<.01]$. As shown in Table 2, when the passage topic was cued, recall of the topic item was higher than recall for the nontopic item. However, if the cue
Table 2

Mean Proportion of Recall About Each Item

\begin{tabular}{clllc}
\hline \multirow{2}{*}{ Cue } & Item of & \multicolumn{2}{c}{ Version } & \\
\cline { 3 - 4 } Topic & Recall & $\mathrm{A}$ & $\mathrm{B}$ & Mean \\
\hline \multirow{3}{*}{ Nontopic } & Topic & .14 & .18 & .16 \\
& Nontopic & .07 & .07 & .07 \\
& Topic & .07 & .13 & .10 \\
& Nontopic & .15 & .07 & .11 \\
\hline
\end{tabular}

Note-The standard error of these means is about .01 .

was not the topic, there was no difference in recall between the topic and nontopic items.

The interaction of rater with cue and item was significant $[F(1,47)=8.59, p<.01]$, but presents no problem in interpretation because the ordering of the cell means for each rater show exactly the same pattern. Furthermore, this interaction accounts for a very small proportion of the variance (eta square $=.11 \%$ ) in contrast to the Cue by Item interaction (eta square $=3.76 \%$ ). Hence, although there were rater effects, they appear to have been unimportant.

Finally, there was a pair of interactions with version that show that the A items and B items differed in their memorability. Version and item interacted $[F(1,47)=$ $29.45, \mathrm{p}<.001]$, as did version, cue, and item $[F(1,47)$ $=6.11, p<.05]$. No other effects approached significance.

\section{Discussion}

The result that information about an item presented first in a passage is recalled better than is information about an item presented later duplicates the finding reported by Meyer (1977) and others that recall is better for the first-mentioned material. However, this result is stronger in this study, since order of appearance in the passage was manipulated separately from the semantic content associated with the item. Thus even though the most important content normally appears first in a passage, initial mention influences recall because it has topic-signaling value in itself.

The results in Table 2, however, contain a more theoretically interesting result, that recall about an item is specific to the relation of the recall cue to the originally perceived passage topic. That is, if the cue is the passage topic, recall favors the topic, but if the cue is not the topic, recall of topic and nontopic information is equal. Marking an item as the passage topic apparently does not govern what the reader stores, since in the nontopic cue case there is no difference in recall of topic and nontopic information. Rather, the original passage topic seems to determine what the effect of a later recall cue will be. Notice that there was no main effect of cue $(p>.1)$, which indicates that the overall level of recall does not depend on whether the cue is the topic. This result conflicts with the memory retrieval role of the passage topic presented above.

There are two problems with these results that motivated the second experiment. The first is that the Item B 
propositions are better recalled overall than Item A propositions ( $13.3 \%$ vs. $8.8 \%$ ), which produced the interactions with version. In contrast, as presented in more detail in Kieras (1980, Note 1), in these passages the $\mathbf{A}$ items are generally preferred to the B items as passage topics. A possible explanation for the difference between the items is that the B items showed a tendency to be more specific than the $A$ items and the facts stated about the B items tended to be more specific and concrete. An example of this difference is the items in the Table 1 passage. Although the A-B label is clearly arbitrary, in practice the passages were composed by starting with an A item and trying to devise a suitable B item; this process apparently resulted in differences in item generality and concreteness. It could be that the more general candidate topic makes the best choice for the passage topic, since it logically subsumes the other possible passage topic, but highly specific facts about a specific item are easier to recall in this type of memory experiment. This problem was explored in the second experiment by using passages for which normative topic choice data were available and comparing passages that showed either a strong bias in favor of one item's being the topic or essentially no such bias.

The second problem is that since the topic-marking manipulation involved a serial position manipulation, the observed effects may be due to simple serial position effects rather than to topic marking. Thus the results could be explained as follows: In the topic cue condition, subjects are able to start their recall with the cued item and then recall the passage sentences in the same order as originally presented. The unmarked item information is recalled poorly because it is recalled last and, so, suffers from output interference. Hence a result like Deese and Kaufman's (1957) primacy-only serial position curve appears. In the nontopic cue condition, the cue encourages the subject to start in the middle of the passage, thus disrupting the normal serial order of recall, leading to poorer performance and making the original version of the passage irrelevant.

The attack on this problem used in Experiment 2 was to include a different passage topic marker, sentence surface subject assignment. This marker would allow different candidate topic items to be marked as topical by surface changes in the sentences, without changing the order of the information in the passage. Also, detailed data were collected on the order of recall, which would allow serial order effects in recall to be directly examined.

\section{EXPERIMENT 2}

In this experiment, passages of three types were used: balanced, biased, and surface subject. The balanced and biased passages appeared in either the A-first or the B-first order. Using topic choice data from earlier studies, the balanced passages were selected to show little or no bias in favor of one of the items, and the biased were chosen to show a strong bias. The surface subject passages appeared in either of two versions. which differed only in which candidate topic appeared as the surface subject of all of the passage sentences. As in Experiment 1, recall was cued with either of the two candidate topics.

\section{Method}

Materials. Using the materials and results from Kieras (1980, 1981), six passages were selected, two of each of the three types: balanced, biased, and surface subject. The candidate topic items of the passages used, together with the proportions of choices of each item and the sample size for these proportions, are shown in Table 3. The balanced and biased passages were chosen from those used in Kieras (1980), in which subjects chose one of the two candidate topics in a forced-choice procedure. The surface subject passages were chosen from those used in Kieras (1981), in which subjects generated noun phrase statements of the passage topics; these were scored for which one of the candidate topics they referred to. Since the statements may have referred to something else, these choices are not exhaustive. For ease in reference here, the label Item A was assigned to the candidate topic that was preferred, even slightly, in each passage.

The balanced passages were chosen to show a symmetrical effect of initial mention on topic choice, with very little bias in favor of one of the items. As can be seen in Table 3, for both balanced passages, there is a strong initial-mention effect and the choices of the two items overall are almost of equal frequency. The biased passages were chosen to show a strong topic bias. The Table 1 example is a biased passage used in this experiment. As shown in Table 1, there is a strong bias in favor of Item A, which in both passages is a very general abstract concept, whereas Item B is a very specific and concrete item. The effect of topicalizing B by initial mention is only to weaken the preference for Item $A$. Finally, the surface subject passages were chosen to show a substantial effect of which candidate topic was marked by appearing as the surface subject of the passage sentences. Table 4 shows one of these passages; Item A is "vaccines," and Item B is "virus." Due to the difficulty of composing such passages, equating the items on salience was not attempted for the work in Kieras (1981); the item choices are thus biased. But notice that there is not an obvious difference in the generality of concreteness of the two items, as is the case in the biased passages. Thus, in the Atopicalized versions of the passages, $A$ was quite the dominant choice for the topic, but in the B-topicalized versions, A and B were chosen roughly equally often. Thus, overall, there is a bias in favor of $\mathbf{A}$.

As in Experiment 1, for the balanced and biased passages, the B-topicalized version consisted simply of the same sentences as the A-topicalized version in a different order. The surface subject passages, however, contained different sentences in the two versions, which conveyed the same propositional content, but which differed in which candidate topic appears in the subject and which in the predicate. All of the passages were five sentences long and were computer justified and printed to occupy about 14 lines. Each passage was printed on a separate page, and the pages were assembled into booklets for each subject. Each subject's booklet contained, first, a page of instructions, followed by six passage pages, followed by a page of arithmetic problems for a distractor task, followed by a page of recall instructions and six recall pages, each containing a cue word that was one of the two candidate topics for one of the passages. The cue words appeared in the same order as the corresponding passages. The order of appearance of the passages in the booklets was separately randomized for each subject's booklet.

Design. The design was between subjects; there were four 
Table 3

Proportion of Choices of Item A and Item B for Each Passage Type

\begin{tabular}{|c|c|c|c|c|c|c|}
\hline \multirow[b]{3}{*}{ Passage Items $(\mathrm{A} / \mathrm{B})$} & \multicolumn{6}{|c|}{ Choice } \\
\hline & \multicolumn{2}{|c|}{ Topicalization Version $\mathrm{A}$} & \multicolumn{2}{|c|}{ Topicalization Version B } & \multicolumn{2}{|c|}{ Overall } \\
\hline & A & B & A & B & A & B \\
\hline & \multicolumn{6}{|c|}{ Balanced $(N=24)$} \\
\hline \multirow[t]{2}{*}{ Isomers/Retinene } & $\begin{array}{l}.83 \\
.79\end{array}$ & $\begin{array}{l}.17 \\
.21\end{array}$ & $\begin{array}{l}.29 \\
.25\end{array}$ & $\begin{array}{l}.71 \\
.75\end{array}$ & $\begin{array}{l}.56 \\
.52\end{array}$ & $\begin{array}{l}.44 \\
.48\end{array}$ \\
\hline & \multicolumn{6}{|c|}{ Biased $(N=48)$} \\
\hline Bioluminescent Light/Flashlight Fish & .83 & .17 & .65 & .35 & .74 & .26 \\
\hline \multirow[t]{2}{*}{ Biotransformation/Liver } & .94 & .06 & .60 & .40 & .77 & .23 \\
\hline & \multicolumn{6}{|c|}{ Surface Subject $(N=11)$} \\
\hline Vaccine/Virus & .91 & .00 & .36 & .27 & .64 & .14 \\
\hline Radio Galaxies/Radio Waves & 1.00 & .00 & .45 & .55 & .72 & .27 \\
\hline
\end{tabular}

Table 4

The A-Topicalized and B-Topicalized Versions of an Example Surface Subject Passage

\section{A-Topicalized Version}

A vaccine is prepared by somehow weakening a virus, of ten by inactivating the DNA and using the protein coat which can be injected to stimulate the immune system of the body. Vaccines require for their preparation the keeping of a large supply of virus that is usually grown in systems such as egg or cell cultures. A vaccine is now under test that may soon control the virus, Hepatitis $B$, that produces a serious and very debilitating disease. Vaccines have become available against many viruses that once caused diseases which were common and of ten incurable although some diseases still cannot be controlled this way. Vaccines were originally discovered and developed by men like Jenner who showed that the virus that causes cowpox, a very mild disease, could prevent infection by the dreaded smallpox.

\section{B-Topicalized Version}

Viruses that have somehow been weakened, of ten by inactivating the DNA and using the protein coat, are used to prepare vaccines which can be injected to stimulate the immune system of the body. Viruses must be kept in large supply in order to prepare vaccines and are usually grown in systems such as egg or cell cultures. The virus, Hepatitis $B$, that produces a serious and very debilitating disease may soon be controlled by a vaccine now under test. Viruses once caused many common and often incurable diseases until vaccines against them became available, although some diseases still cannot be controlled this way. The virus that causes cowpox, a very mild disease, was shown by Jenner, one of the original discoverers and developers of vaccines, to prevent infection by the dreaded smallpox.

groups, each of 15 subjects, one group for each combination of passage version (A topicalized or B topicalized) and recall cue (A or B). Each subject studied the appropriate version of each of the six passages. The design ensured that if a multiple of four subjects was run, each passage appeared equally often in each version-cue combination.

Subjects were assigned to one of the four version-cue combinations in the order that they appeared for the experiment, with each combination used once in each consecutive group of four subjects.

Subjects. Subjects were 60 University of Arizona undergraduates, recruited through campus advertisements, who were paid $\$ 2$ for participating in the 1 th experiment.

Procedure. Subjects were run in groups of one to several people. After reading the instructions on the first page of the booklet, the subjects read each passage in their booklets one at a time. In order to raise the level of recall compared with Experiment 1 and to take advantage of the fewer passages being used, subjects were paced at $2 \mathrm{~min} /$ passage rather than $1 \mathrm{~min} /$ passage as in the first experiment. The instructions asked the subjects to try to remember the facts and ideas in each passage and not to attempt to memorize the exact wording. After completing study of the last passage, the subjects worked the page of arithmetic problems for $8 \mathrm{~min}$. Then the subjects read the recall instructions, and then they began to work on the recall pages in the booklet, which were blank except for a cue word at the top of each page. They were instructed to recall in their own words as much as they could remember about the passage corresponding to the cue word, writing in the space provided on the page. They were paced at $3 \mathrm{~min}$ per recall page and were not allowed to go back.

\section{Results}

Recall. The passages were analyzed into propositions, and the recall protocols were scored for reproduction of each passage proposition, as in Experiment 1. The scoring was done blind with regard to the presented version for the balanced and biased passages, and blind with regard to the recall cue for the surface subject passages. Since the scoring reliability was satisfactory in Experiment 1, a more usual scoring procedure was followed here. Scoring was done independently by two judges, and then a third judge reconciled the two sets of scorings to yield a final single scoring. A strict criterion was used as in Experiment 1. In Experiment 1, propositions were classified as being "about" an item if they were contained in the sentences about each item. A more precise rule was used in this experiment to label each passage proposition according to whether it was about Item A, Item B, or something else. The rule was that a proposition was about an item if it either contained the item (or a synonymous reference to it) as an argument or it embedded or was embedded by a proposition that did contain the item. The few propositions that contained both items were counted as being about both items.

The data were then expressed as proportions of presented propositions about each item that were recalled for each subject in each passage, and these 
were averaged over the two passages within each type. An ANOVA was computed in which the within-subjects factors were passage type (balanced, biased, and surface subject) and item of recall (topic or nontopic). The between-subjects factors were passage version (A topicalized or B topicalized) and cue type (topic or nontopic). Table 5 presents the mean proportions of recall for each combination of passage type, cue, and item of recall. Since there were important version effects only in the biased passages, the table shows averages over versions.

Overall, there was no main effect of version or cue (ps > 1). There was a main effect of passage type: Balanced passages were recalled at .15 , biased at .20 , and surface subject at $.13[\mathrm{~F}(2,112)=8.94, \mathrm{p}<.01]$.

The key results correspond to Experiment 1. Propositions about topic items were recalled better than nontopic items $[18 \%$ vs. $15 \% ; F(1,56)=4.25, p<.05]$. There was an interaction between item of recall and cue $[\mathrm{F}(1,56)=19.91, \mathrm{p}<.01]$. As shown in the last column of Table 5, for the topic cue, the recall of the topic item was superior to the nontopic item, and for the nontopic cue, the recall of the two items was similar. Inspection of Table 5 shows that this pattern clearly differed between the passage types, but the three-way interaction including passage type was not significant $[F(2,112)=1.35, p>.1]$. However, this could be due simply to low power. To clarify the results, separate ANOVAs were computed for each passage type. For the balanced and surface subject passages, the results were comparable to the overall analysis. However, the biased passages were rather different from the other two types. The interaction between item of recall and cue was again significant $[F(1,56)=8.15, p<.01]$, but, as shown in Table 5, it followed a pattern different from that in the other passage types. Inspection of the means for each version, shown in Table 6, shows that the dominant factor in these passages was the difference between the A items, which were the general terms preferred as topic choices, and the specific, but more memorable, B items. Overall, propositions about the B items were recalled much better than those about the $A$ items

Table 5

Mean Proportion of Recall About Each Item for Each Passage Type

\begin{tabular}{llcccc}
\hline & & \multicolumn{4}{c}{ Passage Type } \\
\cline { 3 - 6 } Cue & $\begin{array}{c}\text { Item of } \\
\text { Recall }\end{array}$ & Balanced & Biased & $\begin{array}{c}\text { Surface } \\
\text { Subject }\end{array}$ & Mean \\
\hline \multirow{2}{*}{ Topic } & Topic & .19 & .23 & .17 & .20 \\
& Nontopic & .12 & .12 & .12 & .12 \\
\multirow{2}{*}{ Nontopic } & Topic & .15 & .17 & .12 & .15 \\
& Nontopic & .15 & .24 & .13 & .17 \\
\hline
\end{tabular}

Note-The standard error of these means is about .03 for between-subjects comparisons and about .02 for within-subjects comparisons.
Table 6

Mean Proportion of Recall About Each Item for Biased Passages

\begin{tabular}{clllll}
\hline & \multirow{2}{*}{ Cue } & Item of & \multicolumn{2}{c}{ Version } & \\
\cline { 4 - 5 } Topic & Recall & & $\mathrm{A}$ & $\mathrm{B}$ & Mean \\
\hline \multirow{2}{*}{ Nontopic } & Topic & .20 & .25 & .23 \\
& Nontopic & .26 & .05 & .12 \\
& Topic & .12 & .23 & .17 \\
& Nontopic & .30 & .18 & .24 \\
\hline
\end{tabular}

Note-The standard error of these means is about .04 for between-subjects comparisons and about .03 for within-subjects comparisons.

( $26 \%$ vs. $14 \%$ ). Recall of the B item was always fairly high, but recall of the A item was better when the cue was $A$, such as with the topic cue for the $A$ version or with the nontopic cue in the B version, than when the cue was B (19\% vs. 9\%). Recall of A was also slightly better in the A-first version than in the B-first version (16\% vs. $12 \%$ ). Hence the effect of marking produced by initial mention in the biased passages was present, but it was not very important in determining the pattern of recall compared with the semantic differences between the items.

Order of recall. The order of recall of passage information was scored as follows: For each proposition scored as recalled, the serial number of the subject's sentence containing that proposition was noted. Hence if a subject recalled a certain proposition in his or her third sentence in the recall protocol, that proposition was assigned a recall sentence number of 3 . Protocols containing either no recall or only one sentence of recall for a passage were considered to have undefined order information, and so, they constituted missing data points.

The order of recall proved to be closely related to the order of information in the presented passage. The mean sentence number of recall of each proposition was computed, and the sentence number in the original passage in which the proposition appeared was noted. This yielded a total of 60 data points, consisting of original passage sentence number and mean recall sentence number, for the five original passage sentences, three passage types, two passage versions, and two recall cues. The correlation between sentence of presentation and sentence of recall was high $(r=.843, p<.01)$, suggesting that subjects recalled the passage propositions in essentially the same order as they appeared in the original passage.

To explore the recall order data further, a simple multiple-regression analysis was done to assess the relative importance of version and cue on recall sentence order of each item. The mean recall sentence numbers were converted to a proportionate measure by dividing them by the number of sentences in the recall protocol. A dummy-coded variable, TOPIC, was defined to repre- 
sent whether the item of recall was the item marked as the topic in the passage, and another, CUE, indicated whether the item of recall was cued. The data points were the mean proportionate sentence of recall for each item of recall for each subject in each condition, averaged over passages within types, giving a potential 120 data points per passage type. If the order of recall value was missing for one of the two passages, the value from the other was used as the average value for both. Cases in which the sentence of recall was missing for both passages were not included. A separate forward stepwise regression analysis was done for each passage type.

For the balanced passages ( 74 data points), TOPIC made a significant contribution $\left[\mathrm{R}^{2}=.28 ; \mathrm{F}(1,72)=\right.$ $28.35, \mathrm{p}<.01]$. The contribution of CUE was nonsig. nificant $(p>1)$. For the biased passages ( 80 data points), TOPIC was again significant $\left[\mathrm{R}^{2}=.17 ; \mathrm{F}(1,78)=16.33\right.$, $p<.01]$, and CUE was again nonsignificant $(p>.1)$. Finally, in the surface subject passages, neither TOPIC nor CUE contributed significantly to predicting recall position, which would be expected, because there was no manipulation of sentence order in these passages. These results, together with the simple correlation presented above, argue that recall order is determined primarily by presentation order in the original passage. The recall cue has little or no influence on recall order. Thus subjects recalled a passage in roughly the same order as it originally appeared in, regardless of what was cued.

Finally, the possibility that some of the recall effects noted above could be due to recall order was explored by computing analyses of covariance for each passage type, in which the covariate of the recall of each item was the mean proportionate sentence of recall of each item. The analyses agreed with those reported above on almost all effects, but there were some differences. However, these discrepancies are almost certainly artifactual, since in all but one case the covariate was not significant. Furthermore, there were many missing data points, and the discrepancies appeared in the raw means of the complete cases used. Thus the effects observed on recall appear to be due to the topic and cue factors and not simply products of modifications in recall order.

\section{Discussion}

The main results of Experiment 2 agree with those of Experiment 1: Overall, a topicalized item is recalled better than a nontopicalized one, but this effect depends on the recall cue. A topic cue results in the topic item's being recalled better than the nontopic item, but a nontopic cue results in little difference between recall of the two items. These effects will be discussed further in the general discussion below. The remainder of this section will be devoted to some of the side-products of Experiment 2.

Distinction between topicality and memorability. In the biased passages, the main results did not appear.
Recall of an item was highest if it was the cued item, regardless of which item was topicalized by the passage version. However, the item biased as the topic (Item A) was not recalled better than the nontopic item; the contrary was true. Hence these passages do not produce a cue-topic effect corresponding to the balanced and surface subject passages. The effects observed in Experiment 1 are qualified by the presence of semantic differences between the passage items, but the same effects appear when these differences are eliminated. But the results for the biased passages make the interesting point that topicality and memorability of a passage item are distinct characteristics. The abstract general terms were heavily favored as passage topics in the topic choice data, but the specific concrete items subsumed under these topics were much better recalled. This argues that standing in the passage macrostructure is not the only determinant of recall of a passage proposition.

Simple serial order hypothesis. The hypothesis was presented above that the topicalization and cue effects from Experiment 1 can be explained in terms of output interference resulting from either facilitation or disruption of the subject's strategy of recalling the passage information in the same order as it was originally read. This hypothesis can be criticized as follows: First, under this hypothesis, one would expect that the overall level of recall would be worse under the nontopic cue condition than under the topic condition, since the nontopic cue would always produce disruption of recall, whereas the topic cue would not. However, the average level of recall did not differ between cuing conditions. Second, the same class of effects was observed for the surface subject passages, in which information about the two items was distributed almost uniformly throughout the passage. Finally, the order-of-recall data give little support to the disruption hypothesis: Subjects made little effort to recall the cued item information first, but, rather, they generally tried to recall in the same order as the original passage. Thus the hypothetical disruption of recall order has no clear manifestations, and the observed effects should be attributed to alterations in the perceived topic of the passages, not to disturbances in the order of recall.

Reproduction of passage order. Apparently, subjects can retain much of the original order of information in a passage, as shown by the similarity between input and output order in these results and as observed before by Deese and Kaufman (1957). However, it can be argued that this is not simply verbatim or surface-form memory. For one thing, a strict propositional scoring is very sensitive to near-verbatim recall, because highly paraphrased recall will disagree almost completely with the original text at the level of individual propositions and their arguments under scoring rules of the type used here. This effect is due to the tendency of highly paraphrased recall to not preserve the original embedding structure of the presented propositions. However, if the 
subject retains the syntactical form of the passage sentences, there will be considerable similarity in the embedding structure between the presented and recalled propositions. So, if the recall had much verbatim or surface-form content, the scored level of recall would be considerably higher. Thus the low level observed implies that there is little surface-form reproduction in the recall protocols. Further support for this argument is based on which item appeared as the surface subject of sentences in the recall protocols for the surface subject passages, in which one item was the subject of all of the sentences. Only $37 \%$ of the recall sentences were judged to have the same referent for the surface subject as the original passage sentences had; $21 \%$ had the other candidate topic as the surface subject; and $42 \%$ had some other surface subject. Thus even this simple and distinctive feature of sentence surface structure is not very well retained.

How is the similarity between input and output order explained if there is little surface-level retention of the passage? What readers could actually be remembering is the order of information in the passage macrostructure. That is, the technical passages used in these experiments could have some schema-like properties in which information is presented in some normative order (see Kieras, Note 4, for further discussion). For example, the first sentence about a topic item was usually a fairly general statement and was followed by more detailed information. If the passage macrostructure had a tree-like form that was recalled starting with the general information at the top of the tree and proceeding to the detail information near the bottom of the tree, then the order of output of the propositions would resemble the order of the propositions in the original passage, even without retention of the actual surface form.

\section{GENERAL DISCUSSION}

Both intuition and the memory-location model for topic and cue effects presented at the beginning of this paper would predict that recall would be best overall for topic cues and worst overall for nontopic cues. However, the results contradict this model, because there were no main effects of cue. Hence, in these results, what is being affected is not how much subjects recall from the passage, but rather, what subjects recall. This pattern is clearest in the balanced and surface subject passages in Experiment 2. When the recall cue was the passage topic, the subject favored this topic in his or her recall, but there was some recall about the nontopic item, as well. This argues that the subject has memory access to the item although it was neither marked as the passage topic nor cued. In the nontopic cue condition, the two items seem to be roughly equally well recalled in these passages. Since one item was marked as topical and the other was cued, perhaps subjects could have similar success in recalling them, again implying that equal amounts of information about the two items are in memory.

Hence the effect of topicalization in these passages does not seem to be that of altering how much information was stored about each item. Rather, what could be going on is that the topic marking is an aspect of the passage that the subject stores more or less independently of the propositions retained. If so, then suppose that the reader is asked to recall the passage about a certain item (the cue) and also remembers that this item was marked as the topic of the passage. Then he or she interprets this situation as a task demand that this topical item is more important to recall than the nontopic item, and so he or she favors it in the recall effort. But if the cued item is not the passage topic, the reader feels little need to favor one of the items over the other, and so recall of the two items is roughly equal. Thus the passage topic and recall cue act like instructions to the subject about what portions of the passage he or she should attempt to recall.

This explanation is in conflict with the macrostructure theory presented above, in which topicalization would govern storage priorities. This theory has been supported by many experiments in which information higher in the passage macrostructure was recalled better than information lower in the macrostructure. It seems reasonable to think that a manipulation that alters the topic of the passage would alter its macrostructure and so should change which information would be remembered better. The Britton, Meyer, Simpson, Holdredge, and Curry (1979) and the Kozminsky (1977) studies show just such effects. But there are several differences between the present experiments and those supporting the macrostructure theory. First of all, the usual prose memory experiment is done with a free recall paradigm or with a cue corresponding to the single, unambiguous passage topic. The passages usually used probably have a "tighter" macrostructure than the two-topic passages used here, in that the structure would be organized around one main item and would have a relatively neat tree-like structure. But the two-topic passages used here could be viewed by subjects as having two distinct, but interconnected, macrostructures, one organized around each candidate topic, and one of these marked by the textual surface structure as being more thematically important than the other.

In processing an ordinary passage, the reader could select for storage the most important propositions about the single topic. In processing one of the twotopic passages, the reader could select for storage the most important propositions about each of the two candidate topics and also remember which topic was supposed to be more important. When asked to recall, the reader of an ordinary passage has no problem; any cue to the topic corresponds to the top level of the passage information in memory. But the two-topic passage reader must choose which of the two structures 
to emphasize in recall. The relation of the original topic to the recall cue would be used to make the choice.

If this characterization of the present results is accepted, there are two consequences: One is that the macrostructure theory needs to be modified or expanded to take into account what the reader will do with complex technical passages that do not have an obvious structure organized closely around a single topic. Clearly, some further experiments should be done on the problem. The second consequence is that certain seemingly simple questions about prose memory may not be easily answered. The original question addressed by these studies was simply whether the topic of a passage governs later recall, content difference being controlled. The two-topic passages seemed a good choice as a method to allow altering which item was topical without making serious changes in the passage content. But if readers process such passages completely differently from single-topic passages, the role of the perceived passage topic in the storage and retrieval of prose information cannot be so easily isolated.

\section{REFERENCE NOTES}

1. Kieras, D. E. Initial mention as a cue to the main idea and main item of a technical passage (Tech. Rep. 3). University of Arizona, July 1979.

2. Bovair, S., \& Kieras, D. E. A guide to propositional analysis for research on technical prose (Tech. Rep. 8). University of Arizona, in preparation.

3. Turner, A., \& Greene, E. The construction and use of a propositional text base (Tech. Rep. 63). University of Colorado, Institute for the Study of Intellectual Behavior, April 1977.

4. Kieras, D. E. Abstracting main ideas from technical prose: $A$ preliminary study of six passages (Tech. Rep. 5). University of Arizona, August 1980.

\section{REFERENCES}

Britton, B. K., Meyer, B. J. F., Simpson, R., Holdredge, T. S., \& Curry, C. Effects of the organization of text on memory: Tests of two implications of a selective attention hypothesis. Journal of Experimental Psychology: Human Learning \& Memory, 1979, 5, 496-506.

Clark, H. H., Cohen, J., Smith, J. E. K., \& Keppel, G. Discussion of Wike and Church's comments. Journal of Verbal Learning and Verbal Behavior, 1976, 15, 257-265.
Clements, P. The effects of staging on recall from prose. In R. O. Freedle (Ed.), New directions in discourse processing. Norwood, N.J: Ablex, 1979.

DeEse, J., \& Kaufman, R. A. Serial effects in recall of unorganized and sequentially organized verbal material. Journal of Experimental Psychology, 1957, 54, 180-187.

Johnson, R. E. Recall of prose as a function of the structural importance of the linguistic units. Journal of Verbal Learning and Verbal Behavior, 1970, 9, 12-20.

Kieras, D. E. Initial mention as a signal to thematic content in technical passages. Memory \& Cognition, 1980, 8, 345-353.

Kieras, D. E. The role of major referents and sentence topics in the construction of passage macrostructure. Discourse Processes, 1981, 4, 1-15.

KINTsCH, W. The representation of meaning in memory. Hillsdale, N.J: Erlbaum, 1974.

Kintsch, W. On recalling stories. In M. Just \& P. Carpenter (Eds.), Cognitive processes in comprehension. Hillsdale, N.J: Erlbaum, 1977.

Kintsch, W., \& KeEnan, J. Reading rate and retention as a function of the number of propositions in the base structure of sentences. Cognitive Psychology, 1973, 5, 257-274.

Kintsch, W., Kozminsky, E., Streby, W. J., McKoon, G., \& KeEnan, J. M. Comprehension and recall of text as a function of a content variable. Journal of Verbal Learning and Verbal Behavior, 1975, 14, 196-214.

Kintsch, W., \& van DiJK, T. A. Toward a model of discourse comprehension and production. Psychological Review, 1978, 85, 363-394.

Kozminsky, E. Altering comprehension: The effect of biasing titles on text comprehension. Memory \& Cognition, 1977, 5, 482-490.

MEYER, B. J. F. What is remembered from prose: A function of passage structure. In R. O. Freedle (Ed.), Discourse production and comprehension: Advances in research and theory (Vol. 1). Norwood, N.J: Ablex, 1977.

Perfetti, C. A., \& Goldman, S. R. Thematization and sentence retrieval. Journal of Verbal Learning and Verbal Behavior, $1974,13,70-79$.

Perfetti, C. A., \& Goldman, S. R. Discourse functions of thematization and topicalization. Journal of Psycholinguistic Research, 1975, 4, 257-271.

van DiJK, T. A. Semantic macro-structures and knowledge frames in discourse comprehension. In M. Just \& P. Carpenter (Eds.), Cognitive processes in comprehension. Hillsdale, N.J: Erlbaum, 1977.

van Disk, T. A. Relevance assignment in discourse comprehension. Discourse Processes, 1979, 2, 113-126.

Wike, E. L., \& Church, J. D. Comments on Clark's "The language-as-fixed-effect fallacy." Journal of Verbal Learning and Verbal Behavior, 1976, 15, 249-256.

(Received for publication October 15, 1980; revision accepted May 29, 1981.) 